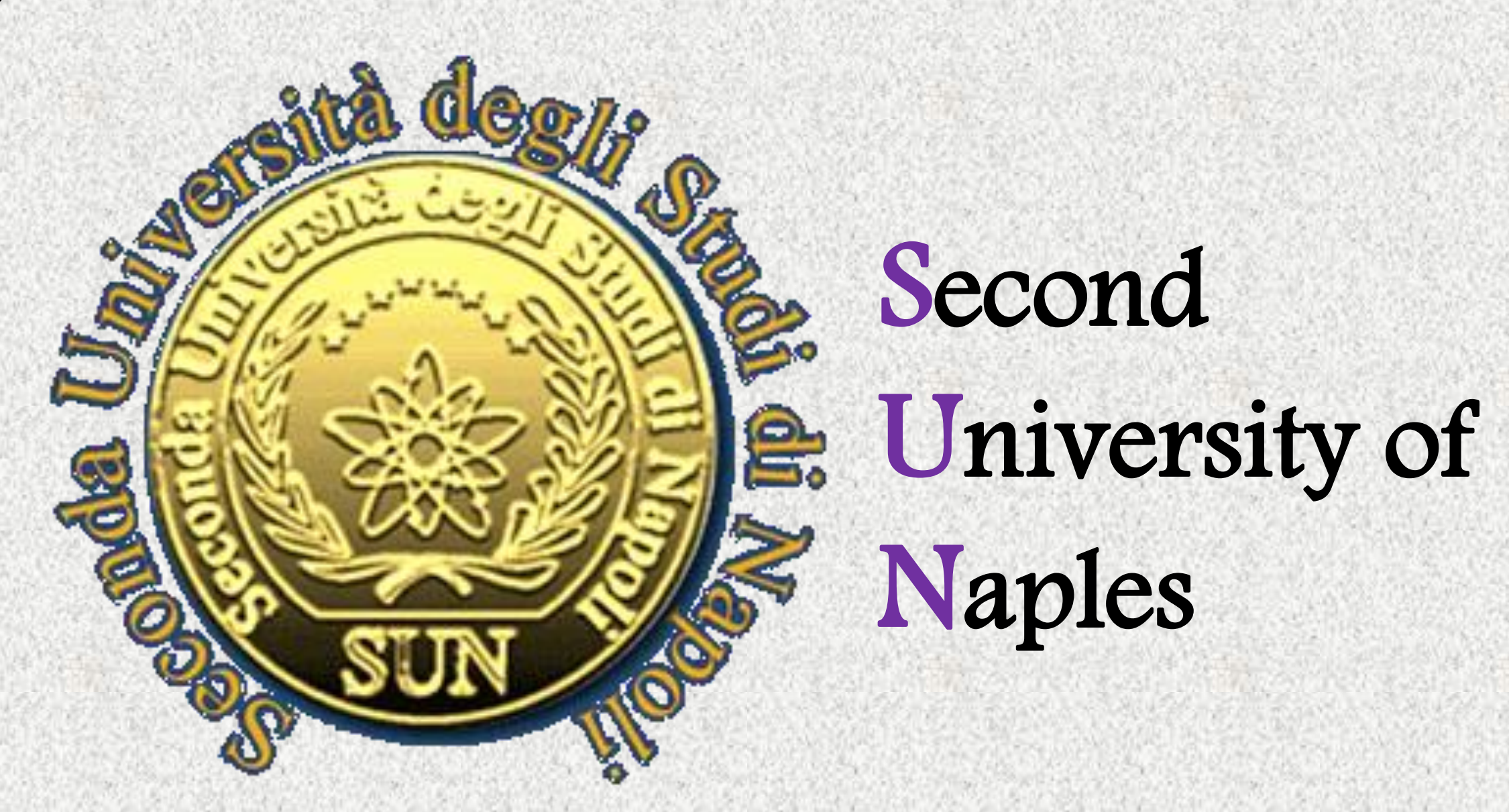

\title{
Rates and reasons for lack of persistence with anti-osteoporotic drugs analysis of the Campania region database
}

Department of Medical and Surgical

Specialties and Dentistry

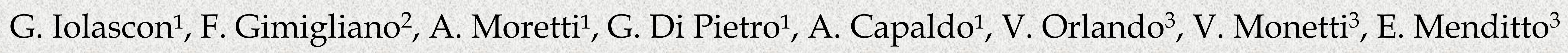

${ }^{1}$ Department of Medical and Surgical Specialties and Dentistry, Second University of Naples, Naples, Italy

${ }^{2}$ Department of Physical and Mental Health and Preventive Medicine, Second University of Naples, Naples, Italy ${ }^{3}$ CIRFF, Center of Pharmacoeconomics, University of Naples Federico II, Naples, Italy

Introduction

Subjects with chronic disorders are more likely to be non-adherent and/or non-persistent to treatment than those with other diseases. Adherence is the extent to which patients take medication as prescribed by their physicians, whereas persistence is the time from treatment initiation to discontinuation.

Low persistence rates to prescribed treatments induce an increased risk of fragility fractures. ${ }^{2,3}$ The most common reasons for discontinuation from anti-osteoporotic medication were: side effects, costs, inconvenient dosing, advice from other specialists, socioeconomic conditions, and lack of motivation. ${ }^{4}$

The aim of our study is to analyze the rates and reasons for discontinuation of anti-osteoporotic drugs in the Campania Region.

We declare that there is no conflict of interest that could be perceived as prejudicing the impartiality of the research project.

\begin{tabular}{|c|c|c|c|c|c|c|}
\hline \multirow[b]{2}{*}{ Time Point } & \multicolumn{2}{|c|}{ Total cohort $(\mathrm{N}=30,048)$} & \multicolumn{2}{|c|}{ Women $(n=28,317)$} & \multicolumn{2}{|l|}{ Men $(n=1,731)$} \\
\hline & Patients on therapy (\%) & $95 \% \mathrm{Cl}$ & Patients on therapy (\%) & $95 \% \mathrm{Cl}$ & Patients on therapy (\%) & $95 \% \mathrm{Cl}$ \\
\hline 3 months & 59.2 & $58.6-59.8$ & 59.4 & $58.8-60.0$ & 55.5 & $53.1-57.8$ \\
\hline 6 months & 34.8 & $34.2-35.4$ & 35.1 & $34.5-35.7$ & 31.1 & $29.0-33.3$ \\
\hline 9 months & 22.3 & $21.9-22.7$ & 22.5 & $22.1-22.9$ & 18.5 & $16.8-20.3$ \\
\hline 1 year & 13.4 & $13.0-13.8$ & 13.5 & $13.1-13.9$ & 10.9 & $9.6-12.3$ \\
\hline
\end{tabular}

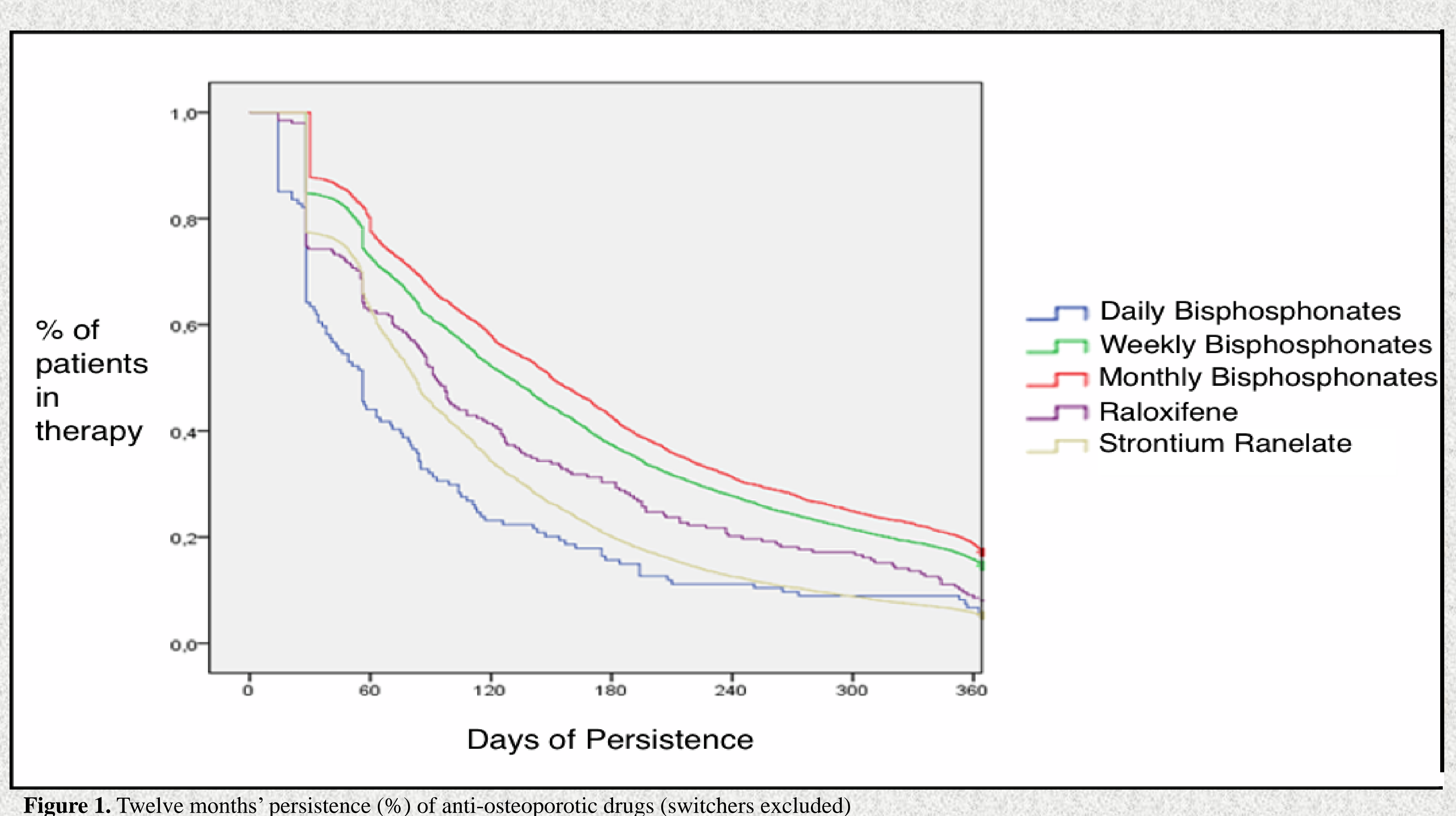

Results

A total of 30,048 were incident users of anti-osteoporotic drugs: 1,731 (5.8\%) males and 28,317 (94.2\%) females. Baseline characteristics of the study population are shown in table 1. Weekly bisphosphonate (BP) $(51.1 \%)$, was the most commonly prescribed drug, followed by strontium ranelate $(30.2 \%)$, monthly $(51.1 \%)$, was the most commonly prescribed drug, followed by stronti.
bisphosphonate $(17.5 \%)$, raloxifene $(0.7 \%)$ and daily bisphosphonate $(0.4 \%)$.

Co-prescription with calcium and vitamin $\mathrm{D}$ was most common for monthly bisphosphonate $(61 \%)$. On the other hand, patients starting with daily bisphosphonate and weekly bisphosphonate were given fewer coother hand, patients starting with daily bisphosphonate and weekly bisphosphonate were given fewer co-
prescriptions of calcium and vitamin $\mathrm{D}(35.8 \%$ and $39.8 \%)$ respectively. In the overall study cohort, 1,532 prescriptions of calcium and vitamin $\mathrm{D}(35.8 \%$ and $39.8 \%)$ respectively. In the overall study cohort, 1,532
$(5.1 \%)$ were switchers. Switching rates were highest for patients taking daily bisphosphonate $(21.6 \%)$ and (5.1\%) were switchers. Switching rates were highest for patients taking daily bisphosphonate $(21.6 \%)$ and lower for patients taking monthly bisphosphonate and weekly bisphosphonate $(6.8 \%$ and $4 \%)$ respectively. In the overall cohort study, persistence rates were evaluated at 90,180, 270 and 365 days after initiation of treatment. In the overall population, $34.8 \%$ of subjects were still on therapy after 6 months. At one year, persistent patients were $13.4 \%$. Kaplan-Meier analysis showed the details grouped by individual regimen (figure 1). At 12 months the number of patients that remained on treatment were: monthly bisphosphonate $17.2 \%$; weekly bisphosphonate $14.7 \%$; raloxifene $8.1 \%$; strontium ranelate $5.4 \%$; daily bisphosphonate $5.2 \%$ log-rank test $(2$ degrees of freedom $p<0.0001)$. (table 2$)$

A multivariate Cox proportional hazard analysis was estimated to identify variables that were significantly associated with non-persistence.(table 3) Patients who were initiated on daily regimen (HR 1.98) and strontium ranelate (HR 1.6) remained at a higher risk of early discontinuation compared to patients initiated with weekly regimen. Male gender was associated with a $11 \%$ higher risk of discontinuation. Patients who starting treatment with a co-prescription with calcium and vitamin D had a lower risk of early discontinuation.

Conclusions

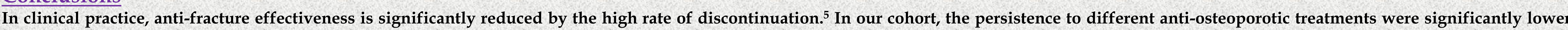

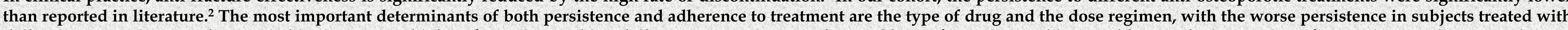
daily BP or strontium ranelate. Switching rates were highest for patients taking daily BP or strontium ranelate and lower for patients taking weekly BP. The integration of strategies to enhance persistence to anti-osteoporotic drugs may have a widespread and economically sustainable impact and could improve treatment effectiveness and clinical outcomes in a real-life scenario. 